\title{
1 Existentialismus in Österreich. Einleitung
}

Jean-Paul Sartres voluminöse phänomenologische Ontologie L'Être et le Néant (1943) zählt zu der Lektüre, die das verzweifelnde Ich in Ingeborg Bachmanns Roman Malina (1971) „betört“"1 zurücklässt. Den delinquenten Jugendlichen in Elfriede Jelineks Die Ausgesperrten (1980) dient sie als Fundus für großspurige Zitate und zur Rechtfertigung ihrer Straftaten. Sartres nobelpreisbefördernde Autobiographie Die Wörter wiederum gehört zu den wenigen Büchern, die Franz-Joseph Murau, Hauptfigur von Thomas Bernhards Opus Magnum Auslöschung (1986), nach eigenen Angaben zweimal lesen würde. AutorInnen wie Andreas Okopenko, Hertha Kräftner, Milo Dor, Peter Turrini, Josef Winkler, Ruth Aspöck, Werner Schwab und Gerhard Roth weisen sich selbst als vom Existentialismus beeinflusst aus, andere beziehen offensiv gegen ihn Stellung, wie Peter Handke, den Sartres Konzept der littérature engagée Mitte der 1960er-Jahre öffentlichkeitswirksam aus der Fassung bringt.

Auf welche Weise und in welcher Gestalt sich der Existentialismus von Paris aus nach 1945 seinen Weg Richtung Österreich bahnt, lässt sich anhand eines Corpus aus Zeitungen, Zeitschriften und Anthologien, Theaterspielplänen und -kritiken, Vorlesungsverzeichnissen und Hochschulschriften sowie Zeugnissen alliierter Kulturaktivitäten mithilfe der Kulturtransfertheorie erfassen. Für die Initiatoren dieses Mitte der 1980er-Jahre am Pariser Centre national de la recherche scientifique begründeten Ansatzes (cf. Kap. 2), Michel Espagne und Michael Werner, ist ein Kulturimport „nie ein rein kumulatives, sondern immer ein schöpferisches Verfahren“,2 das gemäß den Bedürfnissen der Zielkultur Sinnverschiebungen und Funktionsverlagerungen impliziert. Im Unterschied $\mathrm{zu}$ früheren Formen der Einfluss- und Rezeptionsforschung würden auf diese Weise Hierarchisierungen, also ,richtige oder ,falsche' Aufnahmen entfallen; das transferierte Kulturem habe ebenso viel Legitimität wie das Original (,autant de légitimité que l'original“33). Die Fokussierung auf „die in der Rezeptionskultur ausgelösten Verarbeitungsvorgänge“ ${ }^{4}$ steht der in Sartres Qu'est-ce que

1 Ingeborg Bachmann: Malina. Frankfurt am Main ${ }^{1} 1980$, S. 81.

2 Michel Espagne und Michael Werner: Deutsch-französischer Kulturtransfer als Forschungsgegenstand. Eine Problemskizze. In: Espagne und Werner (Hg.): Transferts. Les relations interculturelles dans l'espace franco-allemand (XVIIIe et XIXe siècle). Paris 1988, S. 11-34, hier S. 21.

3 Michel Espagne: La Notion de transfert culturel. In: Revue Sciences/Lettres 2013, Nr. 1, S. 1-9, hier S. 2. http://rsl.revues.org/219 (einges. 09.01.2019).

4 Michael Werner: Dissymmetrien und symmetrische Modellbildungen in der Forschung zum Kulturtransfer. In: Lüsebrink und Reichardt, mit Keilhauer und Nohr (Hg.): Kulturtransfer im Epochenumbruch. Frankreich - Deutschland 1770 bis 1815. (Deutsch-Französische Kulturbibliothek 9.1.) Leipzig 1997, S. 87-101, hier S. 96. 
la littérature? beschriebenen Produktivität von Rezeptionsprozessen nahe, nach der Werke nur in dem Ausmaß Wert haben, in dem sie aufgenommen werden, und dies immer wieder neu, da sich die Lesenden stets en situation befinden. Für den Umgang mit dem Phänomen Existentialismus, das sich ausdrücklich nicht als festumrissenes System verstanden wissen will, sondern als ein fortlaufend zu adaptierendes, im Werden begriffenes Denken, eignet sich die Kulturtransfertheorie durch ihre Situationsgebundenheit und Prozessualität ideal: ebenso wie einzelne Kulturgüter betrachtet sie Kulturen auf der Makroebene nicht als geschlossene Einheiten, sondern als hybride, dynamische Gefüge, als variable „Ensembles von Praktiken, Zeichen und Bedeutungszuschreibungen“"5. Ihr Augenmerk richtet sich speziell auf „Spuren des Fremden in der eigenen Kultur"6 und auf die Frage, wie diese dorthin gelangt sein können, das heißt, nach welchen Kriterien kulturelle Elemente von institutionellen oder privaten Vermittlungsinstanzen selektiert und befördert oder blockiert werden.

Zwar bestimmen die spezifischen Erwartungen der Zielkultur in hohem Maß die Grenzen des Transferierbaren, allerdings ist gerade der sich zwischen 1945 und 1955 vollziehende Existentialismus-Transfer wegen des besatzungsbedingten Machtgefälles nicht von der alliierten Kulturpolitik Frankreichs (cf. Kap. 4.2) ${ }^{7}$ und ihrem Beitrag zur Identitätsbildung der Zweiten Republik zu trennen. Um zu ermessen, worin sich die Strömung nach der Rekontextualisierung von der französischen Ausprägung unterscheidet, beleuchtet Kapitel 3 zunächst den Ausgangskontext: die Entstehung, die VertreterInnen und die Tendenzen des Existentialismus. Das Gewicht liegt - den empirisch erfassbaren Transferinhalten entsprechend - primär auf dem Widerhall, den Jean-Paul Sartre als zentraler Repräsentant findet. Verwandte Ausprägungen existenzphilosophischen Schreibens - von Simone de Beauvoir, Albert Camus, Gabriel Marcel, Emmanuel

5 Werner Suppanz: Transfer, Zirkulation, Blockierung. Überlegungen zum kulturellen Transfer als Überschreiten signifikatorischer Grenzen. In: Celestini und Mitterbauer (Hg.): Verrückte Kulturen. Zur Dynamik kultureller Transfers. (Studien zur Inter- und Multikultur 22.) Tübingen 2003, S. 21-35, hier S. 23.

6 Hans-Jürgen Lüsebrink und Rolf Reichardt: Kulturtransfer im Epochenumbruch. Fragestellungen, methodische Konzepte, Forschungsperspektiven. Einführung. In: Lüsebrink et al. (Hg.): Kulturtransfer im Epochenumbruch, S. 9-26, hier S. 20.

7 Ebenso wenig ist der Transfer isoliert von der Kulturpolitik der anderen Besatzungsmächte zu betrachten, wie sich etwa an amerikanischen Kulturimporten zeigt, die Österreich nicht allein von Seiten der USA erreichen, sondern auch in Form von bereits mit ihnen amalgamierten französischen Kulturimporten (cf. Kap. 5.1). Nach 1950 wirkt sich besonders der die österreichische Besatzungspolitik dominierende Kalte Krieg auf den Transfer der Werke des sich der Sowjetunion annähernden Sartre aus (cf. Kap. 8). 
Mounier und Maurice Merleau-Ponty, die, oft zugeordnet, teils wesentlich von Sartres Positionen abweichen und sich nur bedingt mit dem Etikett Existentialismus identifizieren - werden im Ausmaß ihrer tatsächlichen Rezeption in Österreich berücksichtigt (cf. Kap. 3.1).

Die Untersuchung des Aufnahmekontexts - das soziopolitische Klima und die kulturelle Landschaft in Österreich - macht deutlich, warum Sartre nicht nur in Frankreich zur richtigen Zeit kommt, um mit seinem Freiheitsdenken eine Breitenwirkung zu erzielen, sondern auch in Österreich, wo der literarisch-philosophische Leerstand nach Jahren der Abgetrenntheit vom internationalen Geistesgeschehen erheblich ist. Ein erstes Rezeptionsangebot wird indes fast vollständig ausgeschlagen: Das Résistance-Stück Die Fliegen, das in Westdeutschland als Theater-Ereignis der Saison 1947/48 eine intensive Debatte über die Notwendigkeit von Reue auslöst, wird dort von den französischen Alliierten aktiv gefördert, während man in Österreich, das durch seinen 1943 von der britischen, sowjetischen und amerikanischen Regierung festgelegten Opferstatus (als „the first free country to fall victim to Hitlerite aggression“8 ${ }^{\text {) }}$ von Frankreich als pays ami behandelt wird, auf Kontinuität setzt: Vor allem Klassiker der Habsburgerzeit ohne nennenswertes Polarisierungspotential und ein konformes französisches Angebot sollen die Rückbesinnung auf die eigene kulturelle Größe und somit das Zusammengehörigkeitsgefühl stärken. Zumal im Vergleich zu Deutschland wird im Folgenden eruiert, inwieweit diese unterschiedliche Ausgangslage Auswirkungen auf Art und Umfang der Aufnahme des aus dem Résistance-Umfeld erwachsenen und um den Themenkomplex Verantwortung kreisenden Existentialismus hat (cf. Kap. 3.2).

Vorgestellt werden Sartres Philosophie und Prosa dem österreichischen Publikum zunächst in den nach 1945 zahlreich neu erscheinenden Literatur- und KulturPeriodika wie Plan, Wort und Tat, Wort und Wahrheit, Der Turm oder Tagebuch (cf. Kap. 4.3). In diesem Zusammenhang erweisen sich einzelne Schlüsselfiguren als entscheidend für den Kulturtransfer, wobei es sich überwiegend um zwischen den Kulturen stehende AutorInnen, RedakteurInnen und ÜbersetzerInnen handelt, etwa um MitarbeiterInnen bei alliierten Printmedien (wie Armand Jacob oder Lilly

8 United States Department of State: Declaration on Austria [Moskau, 30. Oktober 1943]. In: Foreign Relations of the United States: Diplomatic Papers, 1943. General, Vol. 1, ed. by William M. Franklin and E. R. Perkins (Washington 1963), S. 761 (Annex 6). Die Konsequenzen dieser besatzungspolitischen Besonderheit werden in weiterer Folge ebenso wie mentalitätsgeschichtliche Erwägungen und die stark katholisch flankierte Rezeption in Österreich (cf. Kap. 7.3) als Gründe dafür diskutiert, dass die österreichische Beschäftigung mit dem Existentialismus in anderen Bahnen verläuft als die westdeutsche und sich daher nicht - wie gelegentlich geschehen - undifferenziert unter eine gesamtdeutschsprachige Aufnahme subsumieren lässt. 
von Sauter) oder um ÖsterreicherInnen, die in das französischsprachige Ausland emigriert sind (wie Jean Améry, Manès Sperber und André Gorz). ${ }^{9}$ Ein Thema, das die Nachkriegsperiodika mit Vehemenz verfolgen, ist das der Verantwortung von SchriftstellerInnen: Vergleichsweise einhellig wird so in katholischen, kommunistischen und avantgardistischen Blättern ein Engagement gefordert, das in seinen Grundzügen mit den literaturtheoretischen Inhalten in Sartres Qu'est-ce que la littérature? übereinstimmt (cf. Kap. 6.4). Die nach dem Krieg zu neuer Aktualität gelangte Frage, ob die alte Sprache zur Abbildung des Erlebten und zum Aufbau des Neuen hinreichen kann oder ob sich durch sie bloß alte Denkmuster perpetuieren, wird von den in sprachskeptischer Tradition stehenden und jüngst auch vom Surrealismus beeinflussten NachkriegsautorInnen lebhaft debattiert. Das in Variationen

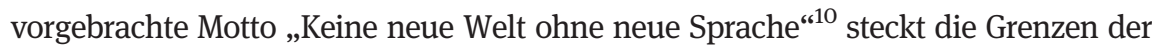
Bereitschaft vieler ab, sich einem auf die Sagbarkeit der Dinge bauenden LiteraturKonzept zu öffnen, das, wenig beeindruckt vom Missverhältnis zwischen Sprache und Realität, ersterer wieder zu Würde und Konstruktivität verhelfen will.

Form und Inhalt der littérature engagée präsentiert das 6. Kapitel, das charakteristische Themen und Motive des Existentialismus als literarische Strömung am Beispiel der neuen österreichischen ErzählerInnen der fünfziger Jahre veranschaulicht. Dabei macht die Literatur der Grenzsituationen (littérature des situations extrêmes) in dem von Hans Weigel herausgegebenen Jahrbuch Stimmen der Gegenwart (1951-1956) deutlich, wie analoge narrative Elemente nicht notwendig im Einfluss des Existentialismus gründen, sondern durchaus Zeitgeist-Ähnlichkeiten geschuldet sein können (cf. Kap. 6.3). Viele Konvergenzen beruhen auf indirekten oder umwegigen Kontakten und Verflechtungen; so führt Franz Kafka, der Einfluss auf das Werk Sartres und Camus' hatte, den jungen österreichischen Kanon nach 1945 an und verstärkt, durch die zahlreichen Anknüpfungspunkte zu seinem existenzorientierten, absurdistischen Schreiben, den Transfer des Existentialismus (cf. Kap. 6.1). Das österreichische Lesepublikum samt den LiteratInnen, die diese Art von Prosa

9 Ohne die Maßstäbe der Zugehörigkeit zur österreichischen Literatur durch Geburtsort, Wohnort oder Selbstverständnis weiter zu diskutieren, bezieht diese Untersuchung neben in Österreich lebenden SchriftstellerInnen (aller Nationalitäten) ÖsterreicherInnen ein, die das Land dauerhaft verlassen haben (etwa Ingeborg Bachmann, Gerhard Rühm), deren Literatur jedoch einen starken Österreich-Bezug aufweist, sowie jene, die während des Nationalsozialismus fliehen mussten, wie der in Österreich-Ungarn geborene Manès Sperber und der nach 1945 als „gelernter Heimatloser“ (Jenseits von Schuld und Sühne. Bewältigungsversuche eines Überwältigten. München ${ }^{2} 1966$, S. 90) ebenfalls nicht nach Österreich zurückkehrende Jean Améry.

10 Ingeborg Bachmann: Das dreißigste Jahr. In: Bachmann: Sämtliche Erzählungen. München ${ }^{8} 2010$, S. 132. 
stark inspiriert, verkörpern allerdings keineswegs den etablierten Literaturbetrieb. Mit dem nach Kriegsende vorherrschenden Eindruck eines Neuanfangs erweisen sich auch der dazugehörige Elan und die literarische Offenheit der ersten Jahre bald als Illusion, an deren Stelle wieder der status quo ante tritt. Es dominiert konservative „Vorkriegsware“11, was dem selbst als Schriftsteller tätigen Kritiker und Publizisten Hans Weigel mehrfach Anlass zur Klage gibt. In Anbetracht des „Vakuums innerhalb unseres geistigen Lebens ${ }^{\text {“12 }}$, bedingt durch die im Exil belassenen AutorInnen, durch die gezielte Förderung von teils belasteten VorkriegsliteratInnen und zuletzt durch das Ignorieren der neuen Generation (cf. Kap. 6.2), verlangt es gerade die jüngeren LeserInnen nach den zu Kriegszeiten unzugänglichen internationalen Neuheiten, unter denen der Existentialismus die aktuellste ist.

Dass der „Bürgerschreck“13 Sartre der österreichischen Jugend zugleich hilft, gegen die geistige Enge der Elterngeneration aufzubegehren, ist ein wesentlicher Erfolgsfaktor, der auch literarisch seinen Niederschlag findet. Um den Preis der inhaltlichen Verflachung erreicht die Strömung in den europäischen Metropolen schnell den Status eines Jugendkults, dessen Maßlosigkeit und Naivität Elfriede Jelineks Roman Die Ausgesperrten (1980) ironisiert. Dass das subversive Potential des Existentialismus eine gewisse Zeitlosigkeit auszeichnet, legt Norbert Gstreins Roman Eine Ahnung vom Anfang von 2013 nahe, der jugendlich-rebellische „Ausflüge ins Existentialistische“"14 in den neunziger Jahren im ländlichen Österreich porträtiert (cf. Kap. 5.2). Als einen für den Transfer unumgänglichen Aspekt blickt Kap. 5.1 zuvor auf die von der Pariser Rive Gauche importierte Mode und den Lebensstil anhand von Zeitungs- und Zeitzeugen-Berichten über ExistentialistInnenTreffpunkte wie das Wiener Kellerlokal „Strohkoffer“, das „Exil“ und das „Café Sport“.

Dass die Kennzeichnung ,existentialistisch“ nicht nur auf eine uneinheitliche Gruppe von Intellektuellen, SchriftstellerInnen und PhilosophInnen ausgedehnt wird, sondern auch auf die im Arrondissement Saint-Germain-des-Prés wirkenden MalerInnen, MusikerInnen und SchauspielerInnen, lässt gerade im

11 Hans Weigel: Brief aus Wien: Zentrum am Rande. In: Der Monat 5 (1952), Nr. 44, S. 179-183, hier S. 180.

12 Hans Weigel: Vorbemerkung. In: Stimmen der Gegenwart, 1951, S. 5.

13 Jean Améry: In die Welt geworfen. Jean-Paul Sartre (1955). In: Améry: Werke, Bd. 4: Charles Bovary, Landarzt. Aufsätze zu Flaubert und Sartre. Hg. von Hanjo Kesting. Stuttgart 2006, S. 189-197, hier S. 196. [Zuerst in: Jean Améry: Karrieren und Köpfe. Zürich 1955.]

14 Norbert Gstrein: Eine Ahnung vom Anfang. München 2013, S. 49 f. 
akademischen Bereich Zweifel an der philosophischen Güte der Denkrichtung aufkommen. So beeinträchtigt der Ruf als skandalumwobene Modeerscheinung die Aufnahme in universitären Kreisen, wie die wissenschaftlichen Publikationen der Jahre 1945 bis 1955 unmissverständlich belegen. Diese werden im 7. Kapitel zusammen mit Lehrveranstaltungen und Hochschulschriften österreichischer Universitäten ausgewertet, wobei „dem Ernst und Engagement des existentiellen Denkens“ ${ }^{15}$ des Dozenten Leo Gabriel - ein vergleichsweise aufgeschlossener Vermittler - besondere Aufmerksamkeit zukommt, ebenso der erheblichen Kritik durch seinen Konterpart Erich Heintel am Institut für Philosophie der Universität Wien, der der Strömung schon vor der Lektüre von Sartres noch nicht übersetztem philosophischem Hauptwerk L'Être et le Néant (1943) jede Wissenschaftlichkeit abspricht (cf. Kap. 7.2).

Generell ist der Existentialismus-Transfer vor dem Hintergrund vorhandener Übersetzungen und Berichterstattungen, also des potentiellen Wissensstands der RezipientInnen zu betrachten. Als wirkungsvollen Träger seiner Philosophie erachtet Sartre das Theater, das gerade in Österreich einen bedeutenden Platz einnimmt, da seine Stücke schon in der Saison 1947/48 auf den Spielplänen stehen, seine Prosa hingegen größtenteils ab 1949/50, Das Sein und das Nichts erst 1952 in deutscher Übersetzung vorliegt. Die Abfolge von Sartres phänomenologischer, existentialistischer, marxistisch und schließlich anarchistisch gefärbter Phase spiegelt sich wenig in der Übersetzungs- und Rezeptionschronologie. Seine Prosa - der Roman La Nausée, der Erzählband Le Mur und der aus L'Âge de raison, Le Sursis, La Mort dans l'âme und dem unvollendet gebliebenen La Dernière Chance bestehende Roman-Zyklus Les Chemins de la liberté - erscheint bis auf den letzten Teil der Trilogie zwischen 1938 und 1945, Jahre bevor die zunächst auf Dramen und philosophische Kurztexte konzentrierte Erstrezeption in Österreich beginnt. Als seine Romane gelesen werden, hat Sartre seinen Schwerpunkt bereits weiter auf philosophische und politische Texte verlagert, schreibt aber bis in die Mitte der sechziger Jahre noch Theaterstücke.

In der österreichischen Aufführungsgeschichte existentialistischer Dramen (cf. Kap. 8) sticht das in antikommunistischem Ruf stehende Les Mains sales aufgrund mehrerer Skandale, die Sartre zweimal persönlich nach Wien führen, heraus: Scheitert das Drama schon Ende 1950 am Wiener Volkstheater an den verhärteten politischen Fronten des Kalten Kriegs, sorgt Sartre aus Angst vor Instrumentalisierung seines Stücks zwei Jahre später selbst für ein Aufführungsverbot

15 Augustinus Karl Wucherer-Huldenfeld: Leo Gabriel (1902-1987). Zur Bedeutung und Tragweite seines Grundgedankens. In: Benedikt et al. (Hg.): Verdrängter Humanismus - verzögerte Aufklärung, Bd. 6: Auf der Suche nach authentischem Philosophieren. Philosophie in Österreich 1951-2000. Wien 2010, S. 622-635, hier S. 623. 
im Wiener Theater am Parkring und interveniert 1954 gleicherweise am Volkstheater, dannjedoch ohne Erfolg. Der Grund für die Selbstzensur 1952 versetzt seine ZeitgenossInnen in Erstaunen: Sartre tritt im Dezember des Jahres bei dem unter Protektion der Sowjetarmee abgehaltenen Wiener „Völkerkongress für den Frieden“ auf, ein für ihn historisches Ereignis, das nach außen hin den Anfang seiner vierjährigen Phase als compagnon de route der Kommunistischen Partei Frankreichs markiert. Diese sich in Wien öffentlich manifestierende Wandlung, zu einem Zeitpunkt, als die meisten der ursprünglich prokommunistischen europäischen Intellektuellen sich in Anbetracht der Straflager-Enthüllungen von der Sowjetunion distanzieren, sorgt für eine Umkehrung der eingespielten Rezeptionsmuster: Die ehedem an ihm interessierte, westlich orientierte Presse reagiert mit Boykott und Befremden auf den politischen Sartre, während sich die KPÖ-nahen Periodika, die zuvor die existentialistische Freiheitsphilosophie als abstrakt-idealistisch und ignorant gegenüber der sozialen Situiertheit des Individuums heftig attackiert hatten, nun begeistert geben. Sartres zweiter Wien-Aufenthalt räumt letzte Unklarheiten über seine Positionierung im kulturellen Kalten Krieg in den Augen der Allgemeinheit aus und besiegelt die Erstrezeption. ${ }^{16}$ Zwar wird seine Nähe zur Kommunistischen Partei mit dem sowjetischen Einmarsch in Ungarn 1956 enden, doch bleibt sie für die Aufnahme seiner Werke im antikommunistisch geprägten Literaturbetrieb der Zweiten Republik lange bestimmend.

Die in dieser Untersuchung vorgenommene Periodisierung der Erstaufnahme umfasst die Hochphase des Transfergeschehens (1947-1952) sowie die Jahre des Ansteigens (1945/46) und des Abflachens (1953/54), fällt folglich ganz in die Zeit der alliierten Besatzung (1945-1955) Österreichs, durch die, begrenzt von der Zäsur des Weltkriegsendes und der Unabhängigkeit Österreichs 1955, relativ einheitliche Rahmenbedingungen gegeben sind. Für die österreichischen AutorInnen, die in dieser Zeit literarisch sozialisiert werden und dem Existentialismus in ihrer Jugend begegnen (in geringem Maß auch später noch in Verbindung mit der 68er-Bewegung), wird das Zeitfenster weiter geöffnet. Der literarische Nachhall in

16 Sartres Wien-Interventionen stellen einen entscheidenden Rezeptionsschritt für den österreichischen Umgang mit dem Autor dar, weshalb hier das Ende der 1945 beginnenden Erstaufnahme auf das Jahr 1955 datiert wird, im Unterschied zu Westdeutschland, für das Mechtild Rahner die Aufnahme auf die Jahre 1945 bis 1949 begrenzt. Cf. Mechthild Rahner: „Tout est neuf ici, tout est à recommencer ... “. Die Rezeption des französischen Existentialismus im kulturellen Feld Westdeutschlands. (Epistemata, Philosophie 142.) Würzburg 1993, S. 305. 
den Folgejahrzehnten zeigt, dass Kulturtransfers kein klar zu umreißendes Vorher und Nachher aufweisen, sondern sich als Prozess mit verschiedenen Etappen realisieren. ${ }^{17}$ Ausblicke auf spätere Rezeptionsschritte lassen erkennen, dass der Existentialismus nicht nur einen wesentlichen Moment in den austro-französischen Kulturbeziehungen der unmittelbaren Nachkriegszeit darstellt, sondern der österreichischen Literatur und Philosophie bis in das 21. Jahrhundert hinein als Inspiration dient.

Zwar herrscht in der Forschung Konsens darüber, dass Frankreich nach 1945 kulturell zu einem zentralen Modernisierungsmodell für Österreich avanciert, doch steht eine umfassende Untersuchung, wie der Existentialismus - als einer der stärksten literarischen und philosophischen Impulse dieser Jahre - aufgenommen wird, noch aus. Als Forschungsliteratur zum Thema im engeren Sinne existiert ein Aufsatz zur Sartre-Rezeption in den französisch besetzten Bundesländern Tirol und Vorarlberg von Sandra Unterweger ${ }^{18}$ und eine Diplomarbeit von Ulrike Dröscher zu den Wiener Aufführungen des Sartreschen Dramas Die schmutzigen Hände. ${ }^{19}$ Sartres Auftritt beim Wiener „Völkerkongress für den Frieden“ wird berücksichtigt in Michael Kraus’ Diplomarbeit „Kultura“. Der Einfluss der sowjetischen Besatzung auf die österreichische Kultur $1945-1955^{20}$ und in Manfred Mugrauers Aufsatz „Eine ,rein kommunistische Angelegenheit‘? Der Wiener ,Völkerkongress für den Frieden“ im Dezember 1952“21. Zudem streifen einige Untersuchungen zur französischen Kulturpolitik in den Besatzungsjahren sowie zu den kulturellen Wechselwirkungen zwischen Frankreich und Österreich in dieser Zeit den

17 Cf. Jürgen Osterhammel: Transferanalyse und Vergleich im Fernverhältnis. In: Kaelble und Schriewer (Hg.): Vergleich und Transfer. Komparatistik in den Sozial-, Geschichts- und Kulturwissenschaften. Frankfurt am Main 2003, S. 439-466, hier S. 464.

18 Cf. Sandra Unterweger: L'Être ou le Néant? Zur Rezeption des französischen Existentialismus in Tirol und Vorarlberg am Beispiel von Jean-Paul Sartre. In: Unterweger, Vorderegger und Zankl (Hg.): Bonjour Autriche. Literatur und Kunst in Tirol und Vorarlberg 1945-1955. (Edition Brenner-Forum 5.) Innsbruck, Wien, Bozen 2010, S. 285-309.

19 Cf. Ulrike Dröscher: Die ehrbare Koexistenz ... ? Zum Konnex von Theater und Politik in den 1950er-Jahren in Österreich am Beispiel von Jean-Paul Sartres „Die schmutzigen Hände“ am Parkringtheater und am Volkstheater Wien. Diplomarbeit, Wien 2002.

20 Cf. Michael Kraus: „Kultura“. Der Einfluss der sowjetischen Besatzung auf die österreichische Kultur 1945-1955. Diplomarbeit, Wien 2008.

21 Cf. Manfred Mugrauer: Eine ,rein kommunistische Angelegenheit‘? Der Wiener ,Völkerkongress für den Frieden` im Dezember 1952. In: Mikosch und Oberkofler (Hg.): Gegen üble Tradition, für revolutionär Neues. Festschrift für Gerhard Oberkofler. Innsbruck, Wien, Bozen 2012, S. 131-156. 
Existentialismus, darunter diejenigen von Friedrich Koja und Otto Pfersmann, ${ }^{22}$ Thomas Angerer und Jacques Le Rider, ${ }^{23}$ Barbara Porpaczy ${ }^{24}$ und Silke Dürnberger. ${ }^{25}$

Hinzu kommen Beiträge, die sich dem Verhältnis einzelner AutorInnen und ihrer Werke zum Existentialismus widmen, primär zu Elfriede Jelinek (cf. Aufsätze von Johann Sonnleitner, ${ }^{26}$ Paul Jandl, ${ }^{27}$ Marlies Janz ${ }^{28}$ und Ulrike Rainer ${ }^{29}$ ). $\mathrm{Zu}$ Thomas Bernhard sind eine Studie von Juliane Werner ${ }^{30}$ und ein Aufsatz von

22 Cf. Friedrich Koja und Otto Pfersmann (Hg.): Frankreich - Österreich. Wechselseitige Wahrnehmung und wechselseitiger Einfluß seit 1918. Wien, Köln, Graz 1994. Cf. besonders den kurzen Exkurs zur österreichischen Existentialismus-Rezeption von Erwin Waldschütz: Wahrnehmung und Rezeption französischer Philosophie in Österreich, S. 182-220.

23 Cf. Thomas Angerer und Jacques Le Rider (Hg.): „Ein Frühling, dem kein Sommer folgte“? Französisch-österreichische Kulturtransfers seit 1945. Wien, Köln, Weimar 1999. Cf. hierin etwa Susanne Albrecht: Von Molière zu Mnouchkine. Französisches Theater auf Wiener Bühnen, S. 151-166.

24 Cf. Barbara Porpaczy: Frankreich - Österreich. 1945-1960. Kulturpolitik und Identität. (Innsbrucker Forschungen zur Zeitgeschichte 18.) Innsbruck, Wien, München, Bozen 2002.

25 Cf. Silke Dürnberger: Entwicklung und Status quo französisch-österreichischer Kulturtransfers im literarhistorischen Kontext. Eine europäische Zweierbeziehung. (Europäische Hochschulschriften XIII/265.) Frankfurt am Main 2002.

26 Cf. Johann Sonnleitner: Existentialismus im Nachkriegsösterreich. Zu Jelineks Roman Die Ausgesperrten. In: Rétif und Sonnleitner (Hg.): Elfriede Jelinek. Sprache, Geschlecht und Herrschaft. (Saarbrücker Beiträge zur Vergleichenden Literatur- und Kulturwissenschaft 35.) Würzburg 2008, S. 79-88.

27 Cf. Paul Jandl: Mythen. Schmutz. Existentialismus. Film. Zu Elfriede Jelineks „Die Ausgesperrten“. In: Jandl und Findeis (Hg.): Landnahme. Der österreichische Roman nach 1980. Wien, Köln 1989, S. 17-30.

28 Cf. Marlies Janz: Mythendestruktion und ,Wissen'. Aspekte der Intertextualität in Elfriede Jelineks Roman „Die Ausgesperrten“. In: Text + Kritik 1993, Nr. 117 („Elfriede Jelinek“), S. 38-50. Cf. auch Janz: Elfriede Jelinek. Stuttgart 1995.

29 Cf. Ulrike Rainer: The Grand Fraud ,Made in Austria': The Economic Miracle, Existentialism, and Private Fascism in Elfriede Jelinek's Die Ausgesperrten. In: Johns und Arens (Hg.): Elfriede Jelinek: Framed by Language. (Studies in Austrian literature, culture, and thought.) Riverside/CA 1994, S. 176-193.

30 Cf. Juliane Werner: Thomas Bernhard und Jean Paul Sartre. (Internationale Forschungen zur Allgemeinen und Vergleichenden Literaturwissenschaft 193.) Berlin 2016. Cf. auch Manfred Mittermayer: Von Montaigne zu Jean-Paul Sartre. Vermutungen zur Intertextualität in Bernhards Auslöschung. In: Hoell und Luehrs-Kaiser (Hg.): Thomas Bernhard. Traditionen und Trabanten. Würzburg 1999, S. 159-173; sowie Joachim Hoell: Der „literarische Realitätenvermittler“. Die „Liegenschaften“ in Thomas Bernhards Roman Auslöschung. Berlin 1995. 
Walter Wagner ${ }^{31}$ hervorzuheben, zu Ingeborg Bachmann ein Beitrag von Dirk Göttsche. ${ }^{32}$ Existentialistischen Philosophemen bei Marlen Haushofer widmen sich Daniela Strigl ${ }^{33}$ und Pamela S. Saur. ${ }^{34}$ Zum Autor Peter Rosei existiert ein Aufsatz von Lutz Ellrich, ${ }^{35}$ zu Gerhard Roth eine Grazer Dissertation von Heidemarie Grill-Fuchs. ${ }^{36}$

In Anbetracht der internationalen Ausstrahlung Sartres (erforscht unter anderem in Studien zu Italien, Japan, der Türkei, Deutschland, den USA und Lateinamerika) ${ }^{37}$ mag das Fehlen einer umfassenden Untersuchung zu Österreich auf die erwähnte, hauptsächlich literaturbetriebsbedingte Subsumierung des österreichischen Feldes in das westdeutsche, aus dem beispielsweise die meisten deutschsprachigen Übersetzungen existentialistischer Werke stammen, zurückzuführen sein. Da die Aufnahme des Existentialismus in der Bundesrepublik Deutschland ebenso wie jene in der Deutschen Demokratischen Republik und in der Schweiz insgesamt jedoch in anderen Bahnen verläuft als die österreichische, werden entsprechende Forschungsergebnisse - von Jürgen Wertheimer, ${ }^{38}$

31 Cf. Walter Wagner: Aspekte des französischen Existenzialismus in Thomas Bernhards Autobiografie. In: Huber, Judex und Mittermayer (Hg.): Thomas Bernhard Jahrbuch 2009/2010. Wien, Köln, Weimar 2011, S. 95-105.

32 Cf. Dirk Göttsche: Deutscher und Französischer Existentialismus im Werk Ingeborg Bachmanns. In: Blasberg und Deiters (Hg.): Denken / Schreiben (in) der Krise - Existentialismus und Literatur. (Kunst und Gesellschaft. Studien zur Kultur im 20. und 21. Jahrhundert 2.) St. Ingbert 2004, S. 369-398. Cf. auch Joachim Eberhardts Eintrag „Existentialphilosophie und Existentialismus“. In: Albrecht und Göttsche (Hg.): Bachmann-Handbuch. Leben - Werk Wirkung. Stuttgart 2002, S. 212-214.

33 Cf. Daniela Strigl: Vertreibung aus dem Paradies. Marlen Haushofers Existentialismus. In: Bosse und Ruthner (Hg.): „Eine geheime Schrift aus diesem Splitterwerk enträtseln ...“. Marlen Haushofers Werk im Kontext. Tübingen, Basel 2000, S. 121-136.

34 Cf. Pamela S. Saur: Marlen Haushofer's Heroines and Existentialism. In: Acta Germanica 36 (2008), S. 9-19.

35 Cf. Lutz Ellrich: Lakonischer Existenzialismus. In: Stepina (Hg.): Advanced Rosei. Wien 2011, S. 47-65.

36 Cf. Heidemarie Grill-Fuchs: „Hölle oder Himmel?“ Der Einfluss von Albert Camus’ und Jean-Paul Sartres Existentialismus auf das Romanwerk von Gerhard Roth. Graz: Universität Graz, Diss. 2011.

37 Cf. für entsprechende Länderanalysen: Alfred Betschart und Juliane Werner (Hg.): Sartre and the International Impact of Existentialism. Cham 2020; sowie Jonathan Judaken und Robert Bernasconi (Hg.): Situating Existentialism. Key Text in Context. New York 2012.

38 Cf. Jürgen Wertheimer: „Une saison en purgatoire“. Aspekte der Sartre-Rezeption. In: Hay (Hg.): Zur literarischen Situation 1945-1949. Kronberg 1977, S. 270-284. 
Hendrik Feindt, ${ }^{39}$ Gerhard Kurz ${ }^{40}$ und insbesondere von Mechtild Rahner ${ }^{41}$ - eher kontrastierend und im Rahmen von Transferaktivitäten aus ,zweiter Hand“ herangezogen.

39 Cf. Hendrik Feindt: Engagement, empathie, distanciation. Reflets de Sartre dans la littérature de langue allemande. Französisch von Evelyne Dengler-Mahé. In: Revue d'esthétique, Hors série, 1991 (Sartre / Barthes), S. 71-78. Feindt zieht für seinen Aufsatz Werke von Elfriede Jelinek, Hubert Fichte, Alfred Andersch, Theodor W. Adorno und Jean Améry heran.

40 Cf. Gerhard Kurz: Nullpunkt, Kahlschlag, tabula rasa. Zum Zusammenhang von Existentialismus und Literatur in der Nachkriegszeit. In: Gethmann-Seifert (Hg.): Philosophie und Poesie, Bd. 2. (Otto Pöggeler zum 60. Geburtstag.) Stuttgart, Bad Cannstatt 1988, S. 308-332.

41 Neben der genannten, auf ihrer Freiburger Dissertation beruhenden Studie Die Rezeption des französischen Existentialismus im kulturellen Feld Westdeutschlands (1993), die sich auf die Aufnahme des Existentialismus in westdeutschen Zeitschriften 1945-1949 konzentriert, cf. auch Rahner: „Tout est neuf ici, tout est à recommencer“. Die Rezeption des französischen Existenzialismus in Deutschland nach 1945. In: Busch und Combrink (Hg.): Doppelleben. Literarische Szenen aus Nachkriegsdeutschland. Göttingen 2009, S. 205-218. Weiters die Aufsätze: Rahner: Selbst- und Fremdwahrnehmungsmuster in der Rezeption des französischen Existentialismus nach 1945 in Deutschland. In: Frankreich-Jahrbuch 1995: Politik, Wirtschaft, Gesellschaft, Geschichte, Kultur (Themenschwerpunkt: Wahrnehmungsmuster zwischen Deutschland und Frankreich). Opladen 1996, S. 113-132; sowie Rahner: Jean-Paul Sartre als Modell des Intellektuellen im kulturellen Feld Nachkriegsdeutschlands. In: Eßbach (Hg.): Welche Modernität? Intellektuellendiskurse zwischen Deutschland und Frankreich im Spannungsfeld nationaler und europäischer Identitätsbilder. Berlin 2000, S. 317-340. Zur Camus-Aufnahme cf. vor allem Franz Rudolf Weller: Aspekte der Camus-Rezeption in Deutschland (West und Ost) nach 1945. Eine kritische Bilanz. In: Jung (Hg.): Albert Camus oder der glückliche Sisyphos - Albert Camus ou Sisyphe heureux. (Deutschland und Frankreich im wissenschaftlichen Dialog 4.) Göttingen 2013, S. 395-444. 